\title{
Intervenção Coronariana Percutânea com Mínima Dose de Contraste em Pacientes de Alto Risco para Injúria Renal Aguda: Relato de Caso
}

\author{
Low Contrast Coronary Percutaneous Intervention in High-Risk \\ Patient for Acute Kidney Disease: A Case Report
}

Ricardo Peixoto Oliveira ${ }^{1}$, Joberto Pinheiro Sena ${ }^{1}$, Bruno Macedo de Aguiar ${ }^{1}$, José Carlos Raimundo Brito ${ }^{1}$

${ }^{1}$ Serviço de Cardiologia Intervencionista do Hospital Santa Izabel; Salvador, Bahia Brasil

Correspondence addresses: Dr. Ricardo Peixoto Oliveira ricardopxoto@msn.com

Received: September 28, 2019

Revised: October 28, 2019

Accepted: October 30, 2019

Published: December $2^{\text {nd }}, 2019$

Data Availability Statement: All relevant data are within the paper and its Supporting Information files.

Funding: This work was the result of authors' initiative. There was no support of research or publication funds.

Competing interests: The authors have declared that no competing interests exist.

\section{Copyright}

(C) 2019 by Santa Casa de Misericórdia da Bahia. All rights reserved.

ISSN: 2526-5563
A doença arterial coronariana é responsável por elevada morbi-mortalidade em todo mundo e a intervenção coronariana percutânea (ICP) é uma dos pilares de tratamento para essa condição. Entretanto, a exposição a meio de contraste está relacionada a aumento de incidência de doença renal aguda (nefropatia induzida pelo contraste - NIC), principalmente em pacientes de alto risco, conferindo pior prognóstico. Nesse cenário, a utilização da estratégia de intervenção coronariana percutânea com uso de mínimo volume de contraste consolida-se como estratégia adequada para essa situação. Relatamos um caso de paciente idoso, com disfunção renal crônica e doença coronariana sintomática, submetido a ICP com a estratégia de low contrast $P C I$.

Palavras-chave: Doença Arterial Coronariana; Contraste; Nefropatia.

Coronary heart disease is a major cause of cardiovascular morbidity and mortality worldwide. Percutaneous coronary intervention (PCI) is one of the major treatment of this condition. Nevertheless, this form of revascularization is associated with higher levels of acute kidney disease (Contrast Kidney Injury), mainly in high-risk patients, with worse prognosis. So, low-contrast PCI is a safe strategy for this situation. We report a case of an elderly patient, with chronic kidney failure and symptomatic coronary heart disease undergoing to low contrast PCI.

$\underline{\text { Keywords: }}$ Coronary Heart Disease; Contast; Kidney Injury.

\section{Relato de Caso}

JSS, 86 anos, hipertenso, portador de doença arterial coronariana crônica, diabetes melito tipo 2, dislipidemia e disfunção renal crônica (clearance de creatinina estimado em $32 \mathrm{~mL} / \mathrm{min}$ ), admitido em outubro/2019 com relato de dispneia aos esforços moderados e desconforto torácico associado a tontura. Apresenta passado de angioplastia coronariana há 13 anos de artéria descendente anterior, ramo diagonalis e ramo marginal da circunflexa, e em uso de bisoprolol, AAS, sinvastatina, ezetimibe, metformina, pioglitazona e trimetazidina. Eletrocardiograma em ritmo sinusal, sem alterações significativas. Ao exame, estado geral preservado, PA 108x60mmHg, FC 62 
bpm, examecardiovascularsemachadosrelevantes. Exames laboratoriais evidenciaram $\mathrm{Hb} 13.2 \mathrm{mg} /$ $\mathrm{dL}$, creatinina $1.6 \mathrm{mg} / \mathrm{dL}$, glicemia controlada. Realizada cintilografia de perfusão miocárdica, evidenciou-se isquemia apical discreta. Optou-se por encaminhamento para coronariografia, quando então apresentou grave lesão no segmento médio da descendente anterior, re-estenose grave do ramo diagonalis e resultado angiográfico mantido na circunflexa. A coronária direita, dominante, exibia placas angiograficamente moderadas.

$\mathrm{O}$ caso foi discutido com médico assistente e família, sendo optado por revascularização percutânea. Submetido a angioplastia de descendente anterior e ramo intermédio com técnica de low constrast PCI, guiado por estudo ultrassonográfico intravascular (USIC) - utilizouse $21 \mathrm{~mL}$ de contraste iso-osmolar (Figura 1). Paciente permaneceu em vigilância na unidade hospitalar por 72 horas, recebendo alta sem queixas, com função renal inalterada e em uso de dupla antiagregação plaquetária e medicamentos de uso regular.

\section{Discussão}

A doença arterial coronariana (DAC) se apresenta como uma das mais frequentes causas de mortalidade cardiovascular e tem elevada morbidade. ${ }^{1}$

O envelhecimento populacional, os hábitos de vida e a alta prevalência de hipertensão,

Figura 1. Coronariografia antes e após intervenção e USIC.

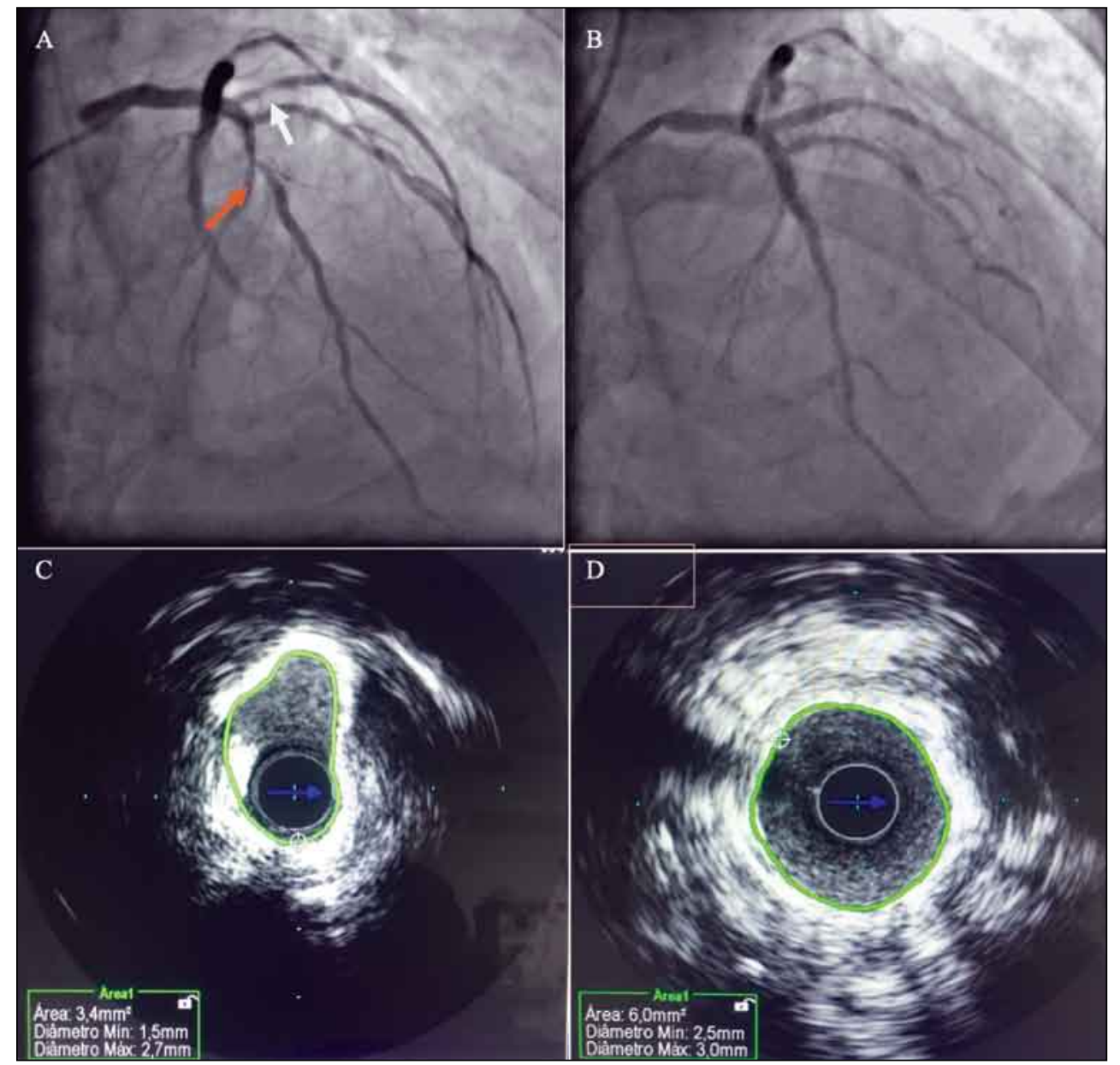

A: grave lesão em descendente anterior (seta laranja) e re-estenose de stent em ramo diagonalis (seta branca). B: resultado angiográfico de controle. C/D: USIC pré e pós-intervenção permitindo avaliar aplaca aterosclerótica, aréa e extensão alcançada, possíveis complicações e aposição do stent. 
diabetes, obesidade e tabagismo estão entre os fatores relacionados com esse achado. Para os pacientes de DAC com anatomia de alto risco ou sintomáticos a despeito de terapia médica otimizada, a ICP é consolidada opção de revascularização em casos selecionados. ${ }^{2}$

Deve-se, no entanto, ressaltar que ao submeter um paciente a estudo angiográfico ou ICP, estar-se-á assumindo um risco de disfunção renal aguda, em graus variados, relacionada a exposição a meio de contraste iodado. A nefropatia relacionada ao contraste, por sua vez, correlaciona-se a maior morbimortalidade. ${ }^{3}$

Define-se nefropatia induzida por contraste (NIC) o cenário no qual existe declínio da função renal após a exposição a contraste iodado, que ocorre em geral 48-72 horas após administração. Dentre as varias definições na literatura, considera-se a ocorrência de um desses achados: ${ }^{4}$

1. Elevação de 1.5 vezes ou mais do valor basal da creatinina em até 07 dias da exposição ao contraste;

2. Aumento do valor sério de creatinina em $0.3 \mathrm{mg} / \mathrm{dL}$ aquém do valor basal dentro de 48 horas após exposição;

3. Débito urinário menor que $0.5 \mathrm{~mL} / \mathrm{Kg} / \mathrm{h}$ por pelo menos 6 horas após exposição.

Os mecanismos relacionados à disfunção relacionada ao uso de contraste não são ainda esclarecidos, mas se postula ocorrência de toxicidade direta a célula tubular ou a efeitos hemodinâmicos relacionados à vascontricção arteriolar e aumento de osmolaridade e viscosidade renal. ${ }^{4,5}$ É necessário considerar como diagnóstico diferencial ateroembolismo, nefrite intersticial aguda e necrose tubular aguda. $^{5}$

A ocorrência de NIC é também influenciada por fatores relacionados ao paciente e ao procedimento (Tabela 1). Os pacientes de maior risco são aqueles com disfunção renal prévia e a ocorrência se acentua quando associada ao diabetes. $\mathrm{O}$ diabetes, por sua vez, parece não estar relacionado a um grande aumento de risco em relação à população geral.

Em contrapartida, reconhece-se na população com disfunção renal crônica, de aumentado risco cardiovascular, uma menor propensão a encaminhamento para estudo angiográfico e revascularização, quando comparado com populações sem essa comorbidade. ${ }^{6}$

Diversas estratégias podem ser utilizadas diante do paciente de risco exposto a contraste iodado. Inúmeras ferramentas de estimativa de risco têm sido validadas para predizer a ocorrência de disfunção renal e racionalizar a abordagem desses pacientes (Figura 2). ${ }^{7}$ Dentre as medidas de nefroproteção, ressalta-se a importância da adequação volêmica (guiada ou não por pressão diastólica final do ventrículo esquerdo), suspensão de drogas nefrotóxicas, uso de contraste de iso-osmolar ou de baixa osmolaridade. $\mathrm{O}$ uso de estatinas vem sendo discutido nesse cenário.

Para os pacientes candidatos a intervenção coronária percutânea, a utilização da técnica de low contrast PCI (ou ultra low constrast PCI), guiando intervenção com USIC demonstrou redução de uso de contraste e ausência de incremento de risco de desfechos maiores, conforme pode se observar no Trial MOZART, que randomizou pacientes para essa estratégia e demonstrou em quatro meses a mesma taxa de eventos e menor uso de contraste. ${ }^{8}$ Considera-se very low contrast $\mathrm{PCI}$ a relação entre volume de contraste e clearance de creatinina menor que 1 $(\mathrm{VC} / \mathrm{Clcr}<1)$, ou zero contrast PCI, quando se dispensa o uso. ${ }^{9}$

A utilização de USIC nesta estratégia é fundamental, pois permite a avaliação anatômica, extensão da lesão, avaliação da placa aterosclerótica, resultado do implante, qualidade da expansão da prótese, bem como complicações, sem utilizar contraste.

Aliado a USIC, a estratégia pode incluir ferramentas no laboratório de hemodinâmica para auxiliar a ICP, tais como: avaliação funcional 
Tabela 1. Fatores de risco relacionados à ocorrência de nefropatia relacionada ao contraste.

\begin{tabular}{ll}
\hline Doença renal crônica & Idade \\
\hline Disfunção ventricular esquerda & Instabilidade hemodinâmica \\
Volume de contraste & Uso de drogas nefrotóxicas \\
Anemia & Hipoalbuminemia \\
\hline
\end{tabular}

Figura 2. Escore de estratificação de risco de NRC.

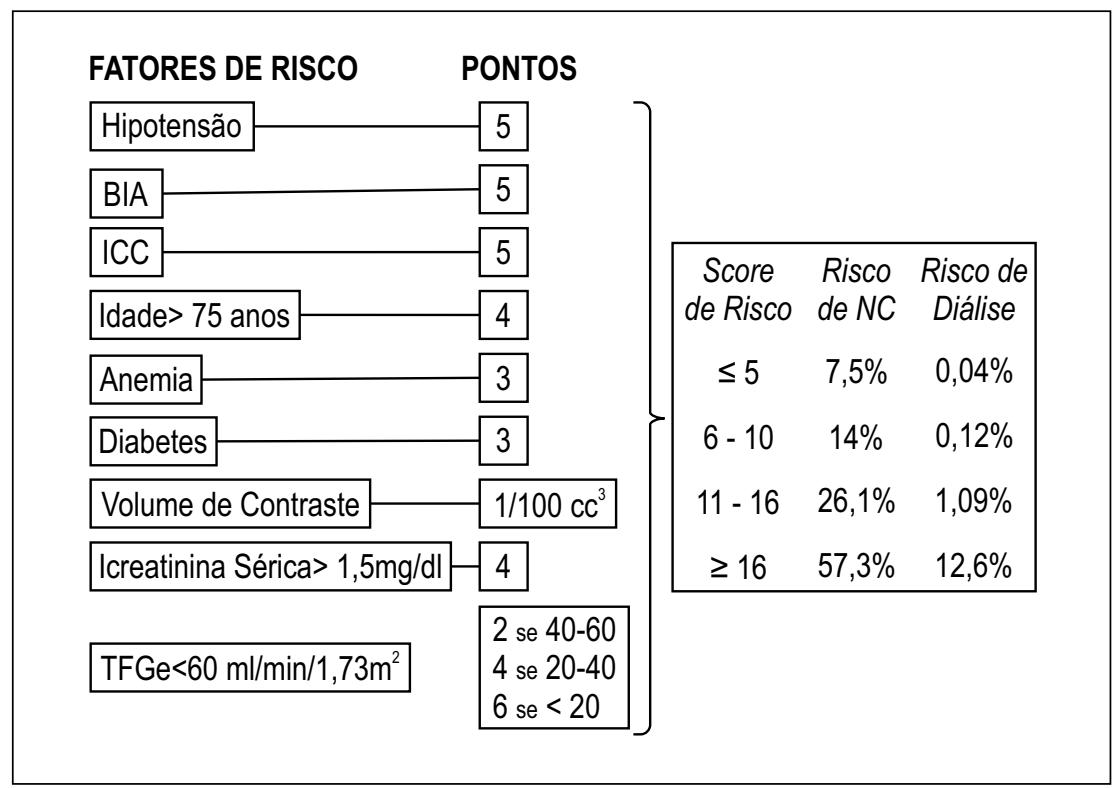

invasiva por exemplo com IFR (Instantaneous Wave Ratio); Roadmap coronário dinâmico, que constrói uma projeção angiográfica digital, dispensando a angiografiaporcontraste(Figura3);

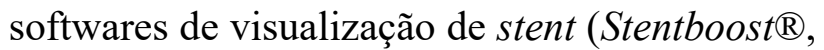
Clear Stent $\mathbb{\circledR}$ ), Dyevert System ${ }^{\circledR}$ (sistema de redução de uso de contraste). É possível também realização de tomografia de coerência óptica sem contraste, utilizando dextran como solução contrastante.

\section{Conclusão}

O tratamento percutâneo da doença arterial coronariana nos dias atuais evoluiu para oferecer segurança e eficácia para pacientes com alto risco de disfunção renal aguda, com pouca ou nenhuma utilização de contraste, sem associar-se a piores desfechos. Diversas ferramentas, integradas,
Figura 3. Roadmap coronário dinâmico: projeção angiográfica digital que permite intervenção sem infusão de contraste.

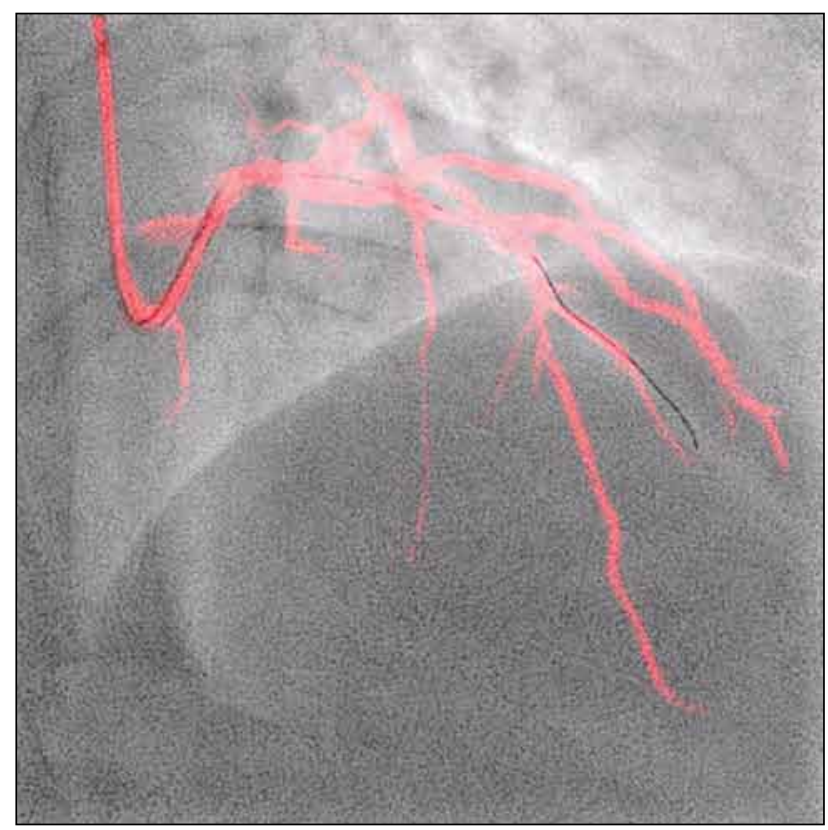


permitem realização de intervenções complexas com mínima ou nenhuma exposição ao contraste. O conhecimento da estratégia de low (ou zero) contrast PCI surge como uma ferramenta que para auxiliar o cardiologista clínico e intervencionista na decisão de estratificação invasiva e revascularização percutânea (quando indicado) a essa população, reconhecidamente de alto risco.

\section{Referências}

1. Roth GA, Johnson C, Abajobir A et al. Global, regional, and national burden of cardiovascular diseases for 10 causes, 1990 to 2015. J Am Coll Cardiol. 2017;70(1):125. doi:10.1016/j.jacc.2017.04.052

2. Knuuti J, Wijns W et al. 2019 ESC Guidelines for the diagnosis and management of chronic coronary syndromes. Eur Heart J. 2019:1-71. doi:10.1093/ eurheartj/ehz425

3. James MT, Samuel SM, Manning MA et al. Contrastinduced acute kidney injury and risk of adverse clinical outcomes after coronary angiography: A systematic review and meta-analysis. Circ Cardiovasc Interv. 2013;6(1):37-43. doi:10.1161/ CIRCINTERVENTIONS.112.974493
4. Mehran R et al. Contrast-associated acute kidney injury. N Engl J Med. 2019;380:2146-55.

5. Santos RO, Malvar B, Silva R et al. Nefropatia de contraste. Acta Med Port. 2011;24(5):809-820.

6. Chertow GM, Normand SLT, McNeil BJ. "Renalism": Inappropriately low rates of coronary angiography in elderly individuals with renal insufficiency. J Am Soc Nephrol. 2004;15(9):2462-2468. doi:10.1097/01. ASN.0000135969.33773.0B

7. Aymong RMED, Nikolsky E, Lasic Z, Iakovou I, Fahy $\mathrm{M}$ et al. A simple risk score for prediction of contrastinduced nephropathy after percutaneous coronary intervention: development and initial validation. J Am Coll Cardiol. 2004;44:1393-1399.

8. Mariani J, Guedes C, Soares P, Zalc S, Campos CM, Lopes $\mathrm{AC}$ et al. Intravascular ultrasound guidance to minimize the use of iodine contrast in percutaneous coronary intervention:the MOZART (Minimizing Contrast Utilization uith IVUS Guidance in Coronary Angioplasty) randomized controlled trial. JACC Cardiovasc Interv. 2014;7(11):1287-1293. doi:10.1016/j.jcin.2014.05.024

9. Kane GC, Doyle BJ, Lerman A, Barsness GW, Best PJ, Rihal CS. Ultra-low contrast volumes reduce rates of contrast-induced nephropathy in patients with chronic kidney disease undergoing coronary angiography. $\mathrm{J}$ Am Coll Cardiol. 2008;51(1):89-90. doi:10.1016/j. jacc.2007.09.019 